\title{
DA HEMODIÁLISE À DIÁLISE PERITONEAL: EXPERIÊNCIAS DE PACIENTES SOBRE A MUDANÇA DE TRATAMENTO
}

\section{FROM HEMODIALYSIS TO PERITONEAL DIALYSIS: PATIENTS' EXPERIENCES REGARIDNG THE CHANGE OF TREATMENT}

\section{DE LA HEMODIÁLISIS A LA DIÁLISIS PERITONEAL: EXPERIENCIAS DE PACIENTES EN EL CAMBIO DE TRATAMIENTO}

\author{
Jeany Freire de Oliveira ${ }^{1}$ \\ Christielle Lidianne Alencar Marinho ${ }^{2}$ \\ Rudval Souza da Silva ${ }^{3}$
}

Como citar este artigo: Oliveira JF, Marinho CLA, Silva RS. Da hemodiálise à diálise peritoneal: experiências de pacientes sobre a mudança de tratamento. Rev baiana enferm. 2019;33:e33818.

\begin{abstract}
Objetivo: conhecer as percepções dos usuários com insuficiência renal crônica que realizavam a diálise peritoneal e vivenciaram previamente a hemodiálise, acerca da mudança de tratamento. Método: estudo exploratório e descritivo, de abordagem qualitativa, realizado com nove pacientes em tratamento com diálise peritoneal. Os dados foram coletados por meio de entrevista semiestruturada, organizados e analisados conforme a Técnica de Análise de Conteúdo Temática. Resultados: da análise das unidades de significado emergiram três categorias temáticas: O medo da mudança de terapia superado pela percepção positiva do tratamento; Ambivalência de sentimentos: independência e autonomia versus aprisionamento; e Alívio dos sintomas físicos. Conclusão: a mudança de tratamento dos usuários com insuficiência renal crônica que realizavam a diálise peritoneal gerou medo do desconhecido, todavia a conquista da autonomia, apesar do sentimento de aprisionamento a uma nova rotina, aliada ao alívio de sintomas físicos, foram experiências reconhecidas como positivas.
\end{abstract}

Descritores: Enfermagem em Nefrologia. Insuficiência Renal Crônica. Diálise Renal.

Objective: to understand the perceptions of users with chronic renal insufficiency who underwent peritoneal dialysis and previously experienced hemodialysis about the change of treatment. Method: exploratory and descriptive study, with qualitative approach, performed with nine patients treated with peritoneal dialysis. Data were collected through a semi-structured interview, organized and analyzed according to the Thematic Content Analysis Technique. Results: the analysis of the meaning units originated three thematic categories: Overcoming the fear of changing the therapy through the positive perception of the treatment; Ambivalence of feelings: independence and autonomy versus imprisonment; and Relief of physical symptoms. Conclusion: the change in the treatment of users with chronic renal

\footnotetext{
Enfermeira. Especialista em Saúde Pública e Vigilância Sanitária. Universidade Federal do Vale do Rio São Francisco. Petrolina, Pernambuco, Brasil. jeanyfroliv@hotmail.com. https://orcid.oro/0000-0002-4181-2326

Enfermeira. Mestre em Hebiatria. Professora da Universidade do Estado da Bahia. Senhor do Bonfim, Bahia, Brasil. https://orcid.org/0000-0002-3827-5494

Enfermeiro. Doutor em Enfermagem. Professor da Universidade do Estado da Bahia. Senhor do Bonfim, Bahia, Brasil. https://orcid.org/0000-0002-799 |-8804
} 
insufficiency who underwent peritoneal dialysis generated fear of the unknown, but the achievement of autonomy, despite the feeling of being trapped to a new routine, allied to the relief of physical symptoms, were recognized as positive experiences.

Descriptors: Nephrology Nursing. Renal Insufficiency, Chronic. Renal Dialysis.

Objetivo: comprender las percepciones de usuarios con insuficiencia renal crónica sometidos a diálisis peritoneal y la hemodiálisis experimentada anteriormente sobre el cambio de tratamiento. Método: estudio exploratorio y descriptivo, de enfoque cualitativo, realizado con nueve pacientes tratados con diálisis peritoneal. Los datos fueron recolectados a través de una entrevista semi-estructurada, organizada y analizada de acuerdo con la Técnica de Análisis de Contenido Temático. Resultados: del análisis de las unidades de significado, emergieron tres categorias temáticas: Temor al cambio de terapia superado por la percepción positiva del tratamiento; Ambivalencia de sentimientos: independencia y autonomía frente a la prisión; y Alivio de los síntomas físicos. Conclusión: el cambio en el tratamiento de usuarios con insuficiencia renal crónica sometidos a diálisis peritoneal genera el miedo a lo desconocido, sin embargo, la conquista de la autonomía, a pesar de la sensación de estar atrapado en una nueva rutina, aliada al alivio de sintomas físicos, fueron reconocidos como experiencias positivas.

Descriptores: Enfermería en Nefrología. Insuficiencia Renal Crónica. Diálisis Renal.

\section{Introdução}

A Doença Renal Crônica (DRC) é um problema de saúde pública decorrente do número crescente de pessoas acometidas. Segundo a Sociedade Brasileira de Nefrologia, que realiza o Censo de Diálise Crônica, houve um aumento de 40 mil novos pacientes em 2017, estimando-se um total de 126.583 pacientes em tratamento dialítico no Brasil naquele ano ${ }^{(1)}$.

Entre os tratamentos adotados, estão a Hemodiálise (HD), o mais difundido no Brasil, a Diálise Peritoneal (DP), ainda pouco utilizada, uma vez que apenas 7,9\% dos pacientes realizavam este tratamento em 2016, e o transplante renal ${ }^{(2)}$. A subutilização da DP não é apenas uma realidade brasileira, pois diversos estudos em outros países apontam uma taxa de adesão abaixo de $10 \%^{(3-7)}$.

Autores asiáticos ${ }^{(4)}$ afirmam que, entre os motivos apontados para a baixa adesão estão a prática predominante de iniciar os pacientes em diálise pela HD e a inércia dos profissionais médicos, dos gestores hospitalares e dos sistemas de saúde ante a implementação de mudanças, haja vista que existem evidências de que a DP pode ser iniciada em tempo hábil e de maneira segura e eficaz em casos urgentes, apresentando bons resultados.

As duas modalidades (HD e DP) de tratamento diferem no modo como afetam a vida dos pacientes. A primeira pode ser muito desgastante para as condições físicas, uma vez que as sessões provocam, dentre outros sintomas e intercorrências, uma fadiga intensa, além de interferir no estilo de vida e nas atividades sociais e laborais $^{(8-9)}$. Já a segunda, a DP, permite ao paciente maior autonomia e flexibilidade temporal e espacial, possibilitando a manutenção das suas atividades diárias ${ }^{(10)}$.

Durante o processo de escolha da modalidade, a decisão geralmente acaba sendo mais influenciada pela opinião dos médicos e a disponibilidade dos métodos do que pelas preferências do paciente, já que nem sempre são disponibilizadas todas as informações acerca dos tipos de diálise e suas implicações na vida diária ${ }^{(8)}$.

A escolha da modalidade de diálise deve ser uma decisão compartilhada entre a equipe de saúde, o paciente e a família ${ }^{(8)}$. Para isso, é preciso garantir que pacientes e familiares sejam esclarecidos sobre os métodos dialíticos disponíveis, para que, assim, sejam conhecidas as suas preferências e respeitado o poder de tomada de decisão pelo paciente na seleção do método. A participação ativa do paciente nesse processo pode contribuir para maior adesão e satisfação com o tratamento.

Diante de tal problemática, surgiu a seguinte pergunta de investigação: Como os pacientes que anteriormente realizavam hemodiálise e 
passaram ao tratamento com diálise peritoneal percebem tal processo de mudança?

No intuito de ampliar as discussões, tentar responder à pergunta e contribuir com o campo de conhecimentos acerca das repercussões diante da permuta entre as duas modalidades nos seus modos de vida, o presente estudo tem por objetivo conhecer as percepções dos usuários com insuficiência renal crônica que realizam a diálise peritoneal e vivenciaram previamente a hemodiálise, acerca da mudança de tratamento.

\section{Método}

Estudo exploratório e descritivo, de abordagem qualitativa, desenvolvido em uma clínica especializada em terapia renal substitutiva, localizada em uma cidade da Região Norte do Piemonte do Itapicuru, interior da Bahia, Brasil. Trata-se de uma clínica de esfera administrativa privada, que fornece serviço ao Sistema Único de Saúde (SUS) e atende 203 pacientes em hemodiálise e 10 pacientes em diálise peritoneal.

Foram considerados critérios de inclusão: pacientes cadastrados no programa de diálise peritoneal e possuir tempo de tratamento na modalidade superior a três meses. E como critérios de exclusão: ter idade inferior a 18 anos, não ter realizado hemodiálise previamente e limitações cognitivas.

A técnica utilizada para obtenção dos dados foi a entrevista semiestruturada, composta por questões que permitiram ao entrevistado expor suas percepções acerca da mudança de terapêutica. Dentre todos os dez pacientes em diálise peritoneal, acompanhados no serviço, nove atenderam aos critérios de inclusão e apenas um não atendeu, por não ter realizado hemodiálise anteriormente.

A coleta de dados ocorreu no serviço, no mês de agosto de 2017, após a consulta mensal, uma vez que a diálise peritoneal é feita em domicílio. As entrevistas foram audiogravadas com o consentimento dos participantes, após todo esclarecimento prestado e assinatura do Termo de Consentimento Livre e Esclarecido (TCLE), e tiveram duração média de 15 minutos. Para garantir o sigilo e o anonimato, os participantes foram identificados com a letra "E", seguida do número da entrevista em ordem cronológica (E1... E9).

As falas foram organizadas e analisadas utilizando-se a Técnica de Análise de Conteúdo Temática ${ }^{(11)}$, que compreende três fases: pré-análise, exploração do material e tratamento dos resultados. Na pré-análise foi realizada a leitura das entrevistas, a fim de apreender os sentidos das falas dos participantes, dando origem às unidades de sentido; durante a exploração do material, ocorreu a separação e o agrupamento das unidades de sentido, de modo a gerar as unidades de significado; e, por fim, após as inferências e interpretação das ideias, emergiram as categorias temáticas.

O estudo foi apreciado e aprovado pelo Comitê de Ética e Pesquisa (CEP), recebendo o Parecer n. 2.182.169, e atendeu às exigências formais que regulamentam as pesquisas envolvendo seres humanos.

\section{Resultados}

Dentre os nove participantes do estudo, sete eram mulheres e dois homens. Quanto ao estado civil, cinco eram solteiros e quatro casadas. Todos possuíam renda de, no máximo, um salário mínimo e não realizavam atividades laborais. Sete encontravam-se na faixa etária de 29-39 anos e dois possuíam mais de 60 anos. Quanto à escolaridade, tratando-se do tempo de estudo, quatro tiveram até oito anos de estudos e cinco estudaram por mais de oito anos.

Em relação ao tempo de tratamento, oito possuíam entre um e cinco anos de diálise peritoneal e apenas um possuía mais de cinco anos. Dois participantes referiram necessitar de ajuda para realizar a diálise em casa; os demais realizavam o autocuidado; oito estavam em Diálise Peritoneal Ambulatorial Contínua (DPAC) e apenas um em Diálise Peritoneal Automatizada (DPA).

Da análise das unidades de significado emergiram três categorias temáticas: Medo da mudança de terapia superado pela percepção positiva do tratamento; Ambivalência de sentimentos: independência e autonomia versus aprisionamento; e Alívio dos sintomas físicos. 
Categoria 1 - Medo da mudança de terapia superado pela percepção positiva do tratamento

Nessa categoria, os entrevistados expressaram o medo relacionado à mudança de terapia, quando surgiu a necessidade de realizar a diálise peritoneal e não mais a hemodiálise, levando-os a acreditar que era uma modalidade mais dolorosa e complicada. Esse sentimento foi causado pelo desconhecimento sobre a nova terapêutica, assim como pela adaptação diante da rotina da hemodiálise:

\begin{abstract}
No começo não aceitei, porque eu tive medo, porque eu não sabia o que era. Eu não entendia nada, não conseguia entender direito. Ai eu fiquei sem querer fazer esse tratamento. Mas teve algumas pessoas assim, que faziam, aí foram me orientando. (E1).

A peritoneal é outra realidade. Se eu soubesse, eu já tinha feito muito antes, porque eu não aceitava. O doutor já queria que eu fizesse há muito tempo, só que eu não aceitava. Eu achava que "ave Maria! carregar esse cateter em mim..." Fazer a cirurgia, eu achava que era coisa de outro mundo. (E3).

Depois que você começa a fazer o tratamento, você vê que não é aquele bicho de sete cabeças que você pensa [...] depois que eu consegui mudar para peritoneal, eu vi que é outra realidade. (E8).
\end{abstract}

\section{Categoria 2 - Ambivalência de sentimentos: independência e autonomia versus aprisionamento}

Após serem questionados sobre as transformações ocorridas na rotina após a mudança de modalidade terapêutica, os participantes relataram maior liberdade para realizar suas atividades diárias e outras atividades ligadas ao lazer, bem como a praticidade, rapidez e autonomia para realizar os procedimentos da diálise.

\footnotetext{
Esse tratamento, eu ainda posso viajar e levar, depende do lugar que eu for, e a hemodiálise não. Na hemodiálise, eu tinha que vir todos os dias, não podia faltar, era pior ainda. (E3).

É rápido, em 20 minutos eu faço, sem dificuldade nenhuma, é manual [...] 15 minutos pro liquido entrar, $e$ 10 minutos pra drenar, é fácil, faço tudo sozinha, não preciso de ninguém, não tenho problemas com isso. (E4).

Com esse agora, faço tudo que quero, faço minhas coisas dentro de casa, que antes não podia fazer [...] arrumo minha casa, cuido de minha filha, e antes não podia fazer, os outros que tinham que fazer por mim [...] e boje, graças a Deus, não sinto dificuldade em fazer nada. (E7).
}

Contudo, os mesmos participantes também relataram um sentimento de aprisionamento relacionado à rotina do tratamento com a diálise peritoneal. Não obstante esta possibilitar mais horas livres no dia, sem a necessidade de permanecerem na clínica regularmente, é uma terapia que requer um rigor tanto em relação aos cuidados de higiene quanto a uma rotina de horários bem estabelecidos para a troca das soluções mais de uma vez ao dia.

É um tratamento que você faz de quatro em quatro horas. Às vezes, você quer ir em um lugar, aí tá ali naquele lugar, às vezes tá bom o ambiente e tal, mas você tem que voltar em casa pra poder fazer o tratamento [...] depois daquelas quatro horas tem que ir embora pra fazer a diálise [...] (E3).

Minha vida se tornou mais presa. Eu não tenbo tempo quase pra nada mais. Tenho que estar fazendo o tratamento sempre nos borários certos, não pode falhar [...] se você tiver um compromisso. não pode ir, tem que fazer $o$ tratamento primeiro, correr atrás da vida, ao invés de ir aos compromissos [...] é bem complicado. (E4).

\section{Categoria 3-Alivio dos sintomas físicos}

No tocante à saúde física, com base nas unidades de significado, é possível que, além dos sintomas provocados pela doença, os pacientes sofram também com aqueles resultantes do tratamento. Com relação a isso, os participantes vivenciaram uma mudança positiva após a diálise peritoneal, uma vez que houve alívio ou desaparecimento de sintomas que eram constantes quando da realização da hemodiálise:

Com esse tratamento, eu me sinto bem melhor. Quando eu estava no começo da doença, que eu fazia bemodiálise antes, sentia uma dorzinha e tinha que parar o que eu estava fazendo. Sentia muita dor e cansaço, e boje não. Hoje não sinto dor, faço a diálise sossegado, não sinto nada. Hoje, graças a Deus, eu estou bem, graças a essa diálise. (E2).

A hemodiálise, "Ave Maria", não quero nem pensar! O melhor pra mim é esse [risos]. A bemodiálise foi a maior tristeza na minha vida, porque eu passava mal, assim [...] passava muito mal, não conseguia comer direito, era muito magrinha, sentia muito frio lá na sala. (E1).

Na peritoneal, você não sente nada, não sente nada, nada mesmo. Eu mesmo não sinto nada [...] na bemodiálise, você passa quatro boras numa máquina que lhe dá dor de cabeça, enjoo, pressão baixa, pressão sobe. Então, a ansiedade de ficar ali também prejudica você. Você fica impaciente, esperando aquelas quatro boras ali, só deitada e fora câimbra, essas coisas, excesso de peso que faz você sentir câimbra [...] (E6). 


\section{Discussão}

O medo da mudança relatado pelos participantes é resultante das incertezas e dos temores acerca do futuro, relacionados ao tempo dispendido, restrições alimentares, esquemas de administração de medicamentos e complicações, como a peritonite, o que também foi observado em outro estudo ${ }^{(12)}$. Além disso, outro estudo ${ }^{(6)}$ com população semelhante mostra que, muitas vezes, os pacientes acabam recebendo informações equivocadas e distorcidas sobre desfechos ruins na diálise peritoneal, uma vez que alguns nefrologistas tendem a recomendar a DP a indivíduos com pior prognóstico, devido à função cardíaca e ausência do acesso venoso.

A descoberta da DRC, por si só, além da dependência por um tratamento para continuar a viver, é um período crítico para qualquer indivíduo, assim como a fase de adaptação à terapia, na qual o paciente apresenta-se fragilizado física e psicologicamente. O tratamento dialítico exige do paciente não só a adoção de um novo estilo de vida, mas também a ressignificação de todas as dimensões do seu existir ${ }^{(13-14)}$.

A HD é a terapia inicial, na maioria dos casos, devido ao diagnóstico tardio e manejo de situações de urgência, em que o paciente inicia a diálise de forma imediata por uma questão de sobrevivência e não de opção. Nesse contexto, há a aceitação do tratamento, mesmo sem os devidos esclarecimentos, diante da situação emergencial, e o paciente geralmente não tem a devida participação no processo decisório nem recebe informações sobre outros tipos de terapia ${ }^{(15)}$.

A escolha do tratamento, teoricamente, é do paciente junto à equipe de saúde, porém isso não tem acontecido na prática, e deve ocorrer não somente no início da terapêutica, mas também quando da necessidade de mudança da modalidade. Estudo ${ }^{(16)}$ sobre a tomada de decisão quanto à seleção da terapêutica, realizado no município de Pelotas (RS), mostrou que a decisão sobre a seleção da Terapia Renal Substitutiva (TRS) foi do profissional médico em 54,2\% dos casos e $83,5 \%$ para os pacientes que realizariam $\mathrm{DP}$ e HD.
O medo gerado pela informação insuficiente, o total desconhecimento acerca da modalidade ou ainda a dificuldade de compreensão sobre cada modalidade terapêutica podem ser apontados como principal causa das recusas iniciais à DP, pois nem sempre os pacientes recebem todas as informações a respeito dos tipos de diálise, bem como sobre a possibilidade de transplante renal ${ }^{(8)}$. Geralmente, a mudança do tipo de terapêutica acontece por conta de necessidade derivada de alterações clínicas no decorrer do tratamento, quando o paciente não possui mais condições de realizar hemodiálise, por falha da modalidade ou problemas na fístula/prótese vascular. Dessa forma, o paciente é comunicado da necessidade de mudança, mas não recebe orientações suficientes sobre a DP, o que o leva a sentir medo, pela falta de conhecimento da nova modalidade, e também frustração, pela falha da anterior ${ }^{(17)}$. Esse fato foi observado nas unidades de significado presentes neste estudo.

Contudo, muitas vezes, durante a consulta, o paciente pode estar abalado emocionalmente e com sua capacidade de entendimento limitada. Dessa forma, detalhes importantes do tratamento podem não ser processados, mesmo que a explicação tenha sido de alta qualidade ${ }^{(5)}$.

Ao se deparar com a DP, o paciente vislumbra um universo completamente novo e desconhecido, no qual se sente sozinho, como se fosse o único a passar pela situação. A busca por informações em diversas fontes passa a ser uma estratégia de enfrentamento. A mais comum dentre elas é ouvir outras pessoas que já realizaram o método, o que não só possibilita conhecer mais sobre o novo como também ajuda a desenvolver maior segurança ${ }^{(18)}$.

A hemodiálise é mais amplamente utilizada e, por isso, pode ser comum os pacientes terem preconceito com outra modalidade. Entretanto, ao serem informados sobre a DP e compreenderem melhor os detalhes da terapia, passam da condição de reféns do medo para um estágio de conhecedores do método e mais otimistas. Desse modo, a informação é libertadora e promotora de autonomia, diminui as angústias, possibilita 
aceitação e faz crer que é possível continuar vivendo com outro tipo de diálise ${ }^{(18)}$.

A troca de modalidade pode ser traumática por gerar mais mudanças no cotidiano, já que o indivíduo precisa passar por um novo processo de adaptação. Esse processo envolve não apenas novos hábitos, comportamentos e uma nova rotina diária como também modificações no domicílio, para que seja compatível com as necessidades da modalidade ${ }^{(17)}$. Passado o momento inicial de crise e com o avançar do processo de adaptação, passam a aceitar a DP, considerando-a um método melhor ao ser comparada à hemodiálise ${ }^{(18)}$.

Cabe destacar que qualquer uma das terapias possui vantagens e desvantagens e que os profissionais de saúde precisam ter habilidades de ensino e comunicação para transmitir informações compreensíveis e de qualidade, utilizar materiais educativos apropriados e desmistificar informações equivocadas, para que o paciente consiga entender o tratamento e promover seu autocuidado $^{(13-14,18-19)}$.

No tocante às mudanças nas atividades cotidianas, estudos ${ }^{(12-13,20)}$ diferentes comparam a DP e a HD quanto à autonomia do paciente e utilizam indicadores como Qualidade de Vida (QV) e níveis de depressão e ansiedade para compreenderem o nível de autonomia. Os resultados apontam que ambas as modalidades afetam a QV. Entretanto, observou-se que, nos pacientes em HD, a qualidade de vida é vista com maiores prejuízos devido à maior restrição quanto à rotina diária. Isso também foi observado nos resultados deste estudo. Já os que realizam a DP podem apresentar melhor $\mathrm{QV}$, por se beneficiarem de maior autonomia e integração social, menos restrições na dieta, maior liberdade para viajar e lidar com assuntos financeiros ${ }^{(5,8,20)}$.

Tratando-se de depressão e ansiedade, são mais frequentes em pacientes em HD, devido à independência restringida, uma vez que esses indivíduos precisam ficar conectados à máquina várias horas por semana e ir ao serviço de diálise a cada dois ou três dias, passando por diversas situações estressantes ${ }^{(8,13,20)}$.
A DP destaca-se entre as TRS disponíveis atualmente, por ser uma modalidade realizada no domić́lio e que possibilita ao usuário uma flexibilidade de horários, permitindo-lhe o gerenciamento e o planejamento de suas atividades diárias ${ }^{(17)}$. Portanto, os pacientes em DP usufruem de maior autonomia, pois são menos dependentes da unidade de diálise e dos profissionais de saúde. Essa modalidade, por ser realizada em domicílio pelo próprio paciente, permite maior atividade no trabalho, maior interação social e a manutenção da rotina habitual ${ }^{(8,21)}$. Essas vantagens são ainda mais evidentes quando a DP é do tipo automatizada, realizada apenas no período da noite, por possibilitar maior tempo livre durante o período diurno ${ }^{(14)}$.

Vale destacar que, apesar de os estudos apontarem essa melhoria da qualidade de vida quando da realização das sessões de DP, os participantes do presente estudo percebiam-se com uma ambivalência de sentimentos com relação à rotina na DP. Enquanto expressaram maior autonomia na modalidade atual, para realizar atividades cotidianas em comparação com a HD, uma vez que não precisariam frequentar a clínica três dias na semana durante quatro horas/dia, sem considerar o tempo de deslocamento para a unidade, os participantes demonstraram insatisfação, por se sentirem aprisionados, devido à obrigação de realizar os procedimentos em horários pré-estabelecidos e mais de uma vez ao dia.

A DP também implica em algumas restrições na rotina, não obstante ser um tratamento realizado em domicílio, que permite maior autonomia em comparação à HD. Dessa forma, o paciente necessita adequar seus hábitos e suas atividades de forma a realizar a diálise com segurança, nos horários corretos ${ }^{(18)}$.

A preocupação com o ritual diário de realização da DP é mais intensa para os pacientes que utilizam a DPAC, pois os procedimentos são realizados manualmente, em média, quatro vezes ao dia, a cada quatro horas. Nessa situação, o indivíduo fica condicionado ao cumprimento rigoroso do tratamento em horários que podem 
coincidir com atividades sociais, de lazer ou trabalho, às quais é obrigado a renunciar ${ }^{(22)}$.

Já na DPA, utiliza-se uma máquina chamada cicladora, que realiza as infusões e drenagens do líquido de diálise durante oito a doze horas à noite, durante o período do sono ${ }^{(22)}$. A DPA é um método de DP que oferece uma liberdade ainda maior, pois o período diurno fica totalmente livre para o desenvolvimento de outras atividades. Por isso, pode oferecer grandes benefícios a esses pacientes que se sentem aprisionados ao tratamento.

Assim, percebe-se que, independente da modalidade do tratamento desenvolvido, são necessários dedicação e comprometimento do paciente em prol do seu autocuidado na convivência com uma doença crônica, reconhecendo que, como toda terapêutica, tem seus pontos positivos e negativos.

$\mathrm{Na}$ última categoria, que tratou dos sintomas físicos, ficou evidenciado que a pessoa que possui a DRC apresenta suas funções orgânicas prejudicadas, em decorrência da uremia, que afeta seu bem-estar geral e sua $\mathrm{QV}^{(14,23)}$. Além dos sintomas provocados pela disfunção renal, há os efeitos das modalidades terapêuticas utilizadas. A HD constitui-se em um tratamento mais desgastante, com técnicas mais agressivas. Por isso, os pacientes apresentam, frequentemente, prurido, câimbras e náuseas, além de fadiga intensa, necessitando de tempo para recuperação após cada sessão ${ }^{(8,19,24)}$.

No presente estudo, as unidades de significado apontam para uma mudança positiva no controle dos sintomas físicos naqueles pacientes que migraram para o tratamento com a diálise peritoneal, uma vez que houve o alívio ou o desaparecimento de sintomas que eram constantes quando da realização da hemodiálise. Outro estudo ${ }^{(16)}$ aponta que pacientes em HD relatam mais dor do que aqueles em DP, justificada pelo posicionamento no decúbito dorsal durante toda a sessão, além das múltiplas punções venosas a cada sessão de HD.

Das modalidades de diálise, a DP é a menos prejudicial no quesito saúde física, ao diminuir a ocorrência desses sintomas e se observarem melhores resultados clínicos e preservação da função renal residual ${ }^{(7,25)}$. Embora os pacientes estejam submetidos ao risco de desenvolver complicações, como a peritonite, o controle sintomatológico é geralmente maior em razão do maior conforto e menor instabilidade hemodinâmica proporcionados pelo método ${ }^{(19,23)}$. Dessa forma, com base nos relatos, é evidente que o alívio dos sintomas físicos é um ponto positivo e contribui tanto com a satisfação do paciente quanto com a adesão ao tratamento.

Como limitações do estudo, podem-se citar o número reduzido de pacientes e a não possibilidade de um outro grupo de pacientes que pudesse ter vivenciado a condição oposta, isto é, ter realizado a DP previamente e permutado pela HD, o que pode ser uma proposta de novos estudos.

\section{Conclusão}

Após a análise das unidades de significado, foi possível identificar as principais percepções acerca da mudança de modalidade para a DP naqueles pacientes que vivenciaram a HD previamente. O medo da mudança devido ao desconhecimento da DP, a conquista de maior autonomia, o sentimento de aprisionamento à rotina da diálise e o alívio de sintomas físicos foram as experiências mais evidenciadas pelos entrevistados.

Vale ressaltar que este estudo não busca exaltar um método em detrimento do outro, já que os dois são extremamente importantes e possuem as suas vantagens. Apenas se destaca que a mudança da HD para a DP foi muito benéfica para esses pacientes.

O estudo das vivências e percepções dos pacientes renais crônicos com as diferentes modalidades de diálise fornece uma importante contribuição para a atuação dos profissionais de saúde, em especial o enfermeiro, em busca de uma atenção à saúde mais integral, e pode subsidiar reflexões acerca da importância de maior esclarecimento quanto às modalidades terapêuticas e o respeito à autonomia do paciente no processo de tomada de decisão. 


\section{Colaborações:}

1 - concepção, projeto, análise e interpretação dos dados: Jeany Freire de Oliveira e Christielle Lidianne Alencar Marinho;

2 - redação do artigo e revisão crítica relevante do conteúdo intelectual: Jeany Freire de Oliveira e Rudval Souza da Silva;

3 - aprovação final da versão a ser publicada: Rudval Souza da Silva.

\section{Referências}

1. Sociedade Brasileira de Nefrologia. Censo de diálise revela 40 mil novos pacientes em 2017 no país. SBN informa [Internet]. 2018 [cited 2018 Mar 13]; 25(114):19-24. Available from: https://arquivos. sbn.org.br/uploads/sbninforma114-2.pdf

2. Sesso RC, Lopes AA, Thomé FS, Lugon JR, Martins CT. Brazilian Chronic Dialysis Survey 2016. J Bras Nefrol. 2017;39(3):261-6. DOI: http:// dx.doi.org/10.5935/0101-2800.20170049

3. Jaar BG, Gimenez LF. Dialysis Modality Survival Comparison: Time to End the Debate, It's a Tie. Am J Kidney Dis. 2018 Mar;71(3):309-11. DOI: 10.1053/j.ajkd.2017.10.022

4. Subramanian S, Javaid MM. Peritoneal Dialysis as the Dialysis Initial Modality of Choice for Renal Replacement Therapy Initiation: Moving from "Why" to "Why Not". Blood Purif. 2017;44:106-7. DOI: $10.1159 / 000475459$

5. Robinski M, Mau W, Wienke A, Girndt M. The Choice of Renal Replacement Therapy (CORETH) project: dialysis patients' psychosocial characteristics and treatment satisfaction. Nephrol Dial Transplant. 2017 Feb;32(2):315-24. DOI: $10.1093 / \mathrm{ndt} / \mathrm{gfv} 464$

6. Yang F, Khin LW, Lau T, Chua HR, Vathsala A, Lee E, et al. Hemodialysis versus Peritoneal Dialysis: A Comparison of Survival Outcomes in South-East Asian Patients with End-Stage Renal Disease. PLoS One. 2015 Oct;10(10):e0140195. DOI: 10.1371/journal.pone.0140195

7. Yang F, Lau T, Luo N. Cost-effectiveness of haemodialysis and peritoneal dialysis for patients with end-stage renal disease in Singapore. Nephrology (Carlton). 2016 Aug;21(8):669-77. DOI: $10.1111 /$ nep. 12668
8. Gonçalves FA, Dalosso IF, Borba JMC, Bucaneve J, Valerio NMP, Okamoto CT, et al. Quality of life in chronic renal patients on hemodialysis or peritoneal dialysis: a comparative study in a referral service of Curitiba - PR. J Bras Nefrol. 2015 Oct-Dec;37(4):467-74. DOI: 10.5935/0101-2800.20150074

9. Medeiros RC, Sousa MNA, Nunes RMV, Costa TS, Moraes JC, Diniz MB. Qualidade de vida relacionada à saúde de indivíduos em hemodiálise. Rev enferm UFPE. 2015;9(Suppl 9):1018-27. DOI: 10.5205/ reuol.8808-76748-1-SM.SM.0909supl201513

10. Machado GRG, Pinhati FR. Tratamento de diálise em pacientes com insuficiência renal crônica. Cad UniFOA. 2014;(26):137-48.

11. Bardin L. Análise de conteúdo. Lisboa (PT): Edições 70; 2015.

12. Roxo NE, Barata RC. Relação Diádica e Qualidade de Vida de Pacientes com Doença Renal Crônica. J Bras Nefrol. 2015;37(3):315-22. DOI: http:// dx.doi.org/10.5935/0101-2800.20150051

13. Reis RP, Lima AP, Laurentino MNB, Bezerra DG. Qualidade de vida e autocuidado do paciente em diálise peritoneal comparado com a hemodiálise: revisão de literatura. Estácio Saúde. 2016;5(2):91-106.

14. Freire XA, Mendonça AEO. Qualidade de vida de pacientes em hemodiálise e diálise peritoneal. Rev Bras Pesq Saúde. 2013;15(4):130-6. DOI: https://doi.org/10.21722/rbps.v15i4.7610

15. Duarte L, Hartmann SP. A autonomia do paciente com doença renal crônica: percepções do paciente e da equipe de saúde. Rev SBPH. 2018;21(1):92-111.

16. Ramos ECC, Santos IS, Zanini RV, Ramos JMG. Qualidade de vida de pacientes renais crônicos em diálise peritoneal e hemodiálise. J Bras Nefrol. 2015;37(3):297-305. DOI: 10.5935/0101-2800. 20150049

17. Schmitz J, Bolfe ER, Costa WCS. Itinerário terapêutico e critérios para escolha da diálise peritoneal em pacientes com doença renal crônica. R Interd. 2018;11(2):17-27.

18. Santos FK, Valadares GV. Conhecendo as estratégias de ação e interação utilizadas pelos clientes para o enfrentamento da diálise peritoneal. Esc Anna Nery. 2013;17(3):423-31. DOI: http:// dx.doi.org/10.1590/S1414-81452013000300004

19. García-Llana H, Remor E, Selgas R. Adherence to treatment, emotional state and quality of life in 
patients with end-stage renal disease undergoing dialysis. Psicothema. 2013 Feb;25(1):79-86. DOI: 10.7334/psicothema2012.96

20. Stasiak CES, Bazan KS, Kuss RS, Schuinski AFM, Baroni G. Prevalência de ansiedade e depressão e suas comorbidades em pacientes com doença renal crônica em hemodiálise e diálise peritoneal. J Bras Nefrol. 2014;36(3):325-31. DOI: http:// dx.doi.org/10.5935/0101-2800.20140047

21. Nowak Z, Laudański K. The perception of the Illness with Subsequent Outcome Measure in More Favorable in Continuos Peritoneal Dialysis vs Hemodialysis in the Framework of Appraisal Model of Stress. Int J Med Sci. 2014 Feb;11(3):291-7. DOI: $10.7150 /$ ijms. 5431

22. Timm AMB, Beuter M, Girardon-Perlini NMO, Pauletto MR, Santos NO, Bruinsma JL. Liberdade condicionada: repercussões na família ao conviver com um membro em diálise peritoneal. Rev Rene. 2015;16(4):540-8. DOI: $10.15253 / 2175-6783.2015000400011$
23. Moura Neto JA, Souza AFP, Moura DQ, Oliveira GM, Paschoalin SP, Paschoalim EL, et al. Modalidade de terapia renal substitutiva como preditora de sintomas depressivos. J bras psiquiatr. 2014;63(4):354-9. DOI: http://dx.doi. org/10.1590/0047-2085000000045

24. Atapour A, Nasr S, Boroujeni AM, Taheri D, Dolatkhah S. A Comparison of the Quality of Life of the Patients Undergoing Hemodialysis versus Peritoneal Dialysis and its Correlation to the Quality of Dialysis. Saudi J Kidney Dis Transpl. 2016 Mar;27(2):270-80. DOI: 10.4103/1319-2442.178259

25. Robinson BM, Akizawa T, Jager KJ, Kerr PG, Saran R, Pisoni RL. Factors affecting outcomes in patients reaching end-stage kidney disease worldwide: differences in access to renal replacement therapy, modality use, and haemodialysis practices. Lancet. 2016 Jul;388(10041): 294-306. DOI: 10.1016/S0140-6736(16)30448-2

Recebido: 9 de outubro de 2019 Aprovado: 8 de novembro de 2019

Publicado: 17 de fevereiro de 2020

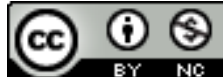

A Revista Baiana de Enfermagem utiliza a Licença Creative Commons - Atribuição-NãoComercial 4.0 Internacional. https://creativecommons.org/licenses/by-nc/4.0/ Este artigo é de acesso aberto distribuído sob os termos da Licença Creative Commons (CC BY-NC). Esta licença permite que outros remixem, adaptem e criem a partir do seu trabalho para fins não comerciais. Embora os novos trabalhos tenham de lhe atribuir o devido crédito e não possam ser usados para fins comerciais, os usuários não têm de licenciar esses trabalhos derivados sob os mesmos termos. 